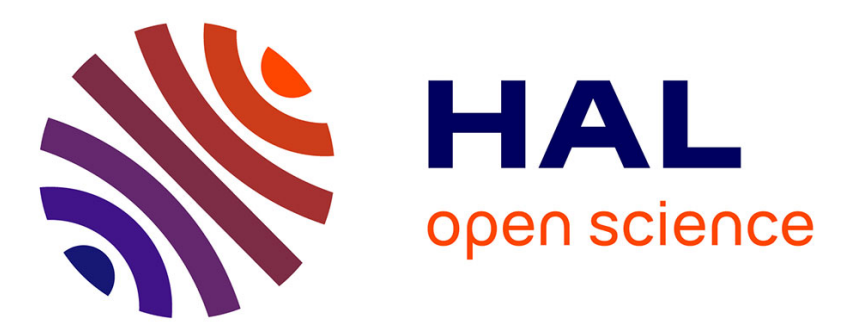

\title{
Voronoi based decentralized coverage problem: from optimal control to Model Predictive Control
}

\author{
Minh Tri Nguyen, Cristina Stoica Maniu
}

\section{To cite this version:}

Minh Tri Nguyen, Cristina Stoica Maniu. Voronoi based decentralized coverage problem: from optimal control to Model Predictive Control. 24th Mediterranean Conference on Control and Automation (MED), Jun 2016, Athens, Greece. pp.1307-1312, 10.1109/MED.2016.7535958 . hal-01390089

\section{HAL Id: hal-01390089 \\ https://hal.science/hal-01390089}

Submitted on 16 Mar 2020

HAL is a multi-disciplinary open access archive for the deposit and dissemination of scientific research documents, whether they are published or not. The documents may come from teaching and research institutions in France or abroad, or from public or private research centers.
L'archive ouverte pluridisciplinaire HAL, est destinée au dépôt et à la diffusion de documents scientifiques de niveau recherche, publiés ou non, émanant des établissements d'enseignement et de recherche français ou étrangers, des laboratoires publics ou privés. 


\title{
Voronoi based decentralized coverage problem: from optimal control to Model Predictive Control
}

\author{
Minh Tri Nguyen, Cristina Stoica Maniu
}

\begin{abstract}
This paper presents a novel decentralized framework for the Multi-Agent dynamical coverage problem subject to anti-collision constraints. The control objective is to authorize each agent operating strictly in its safety zone and then enhance the coverage. These zones are the result of a spatial Voronoi partition of the common working space of the Multi-Agent system based on the current positions of the agents. Each zone provides the local information to design the control policies that make each agent converging to a fixed point inside its Voronoi cell. The performance/effectiveness of the proposed techniques will be demonstrated via numerical examples.
\end{abstract}

Index Terms-Multi-agent dynamical systems, set-theoretic tools, decentralized control, Voronoi partition, coverage problem, optimal control, Model Predictive Control.

\section{INTRODUCTION}

Multi-Agent System (MAS) recently received considerable attention due to the need to manage a group of relatively independent sub-systems for the purpose of achieving a common goal in the same environment. One of the most attractive research field of MAS applications is the collision avoidance (e.g. [1], [2]), because the collisions with obstacles or with other agents are considered as typical faults in most of MAS applications such as traffic control, spacecraft formation flight, etc.

In the last decade, set-theoretic and optimization tools have been employed widely to design the control for MAS subject to anti-collision constraints, notable [3], [4], [5]. In these works, the constraints are described such that the sets characterizing each agent/obstacle do not overlap, therefore the nature of such constraints is non-convex. The design of linear feedback control subject to such constraints using settheoretic methods is introduced in [3].

Traditionally, most of the MAS problems can be solved via a centralized approach. However, because of the limited computation efficiency, decentralized approaches (e.g. [6], [7], [8]) become more appropriate. Paper [8] proposes a set-based decentralized feedback control strategy for MAS, which keeps each agent operating strictly in its safe functioning zone, offering collision avoidance guarantees.

Furthermore, in several Multi-Agent deployment applications, such as rescue operations or surveillance (e.g. [9]), a group of agents have to obtain an optimal coverage over a bounded region w.r.t given constraints.

M.T. Nguyen and C. Stoica Maniu are with Laboratoire des Signaux et Systèmes, CentraleSupélec-CNRS-Univ. ParisSud, Université Paris Saclay, 3 rue Joliot Curie, F-91192, Gifsur-Yvette cedex, France (e-mail: \{minhtri.nguyen; cristina.stoica\}@supelec.fr).
In this context, the main contribution of the present paper is to revisit the coverage control problem subject to the anticollision constraints. These safety constraints can be satisfied by keeping each agent strictly inside a region called functioning zone. These zones are the results of a real time partition step based on the current state (e.g. position) information of the agents. The Voronoi algorithm is employed in this step to decompose the working space into an union of Voronoi cells. After, each agent is associated with one cell which will provide the vertices used to design the control action in order to make each agent converging to a fixed point inside its Voronoi cell. This control design step (based on both the agent's position and the vertices of its Voronoi cell containing the agent) by using the classical framework of optimal control and Model Predictive Control (MPC) is the main contribution of the present paper.

This paper is organized as follows. Section II presents some useful preliminaries and formulates the problem. The local control design for one agent with its associated convex polyhedral functioning zone will be detailed in Section III. In Section IV, we reformulate the previous control in terms of MPC standards. Finally, numerical simulation results are illustrated and discussed in Section V, followed by concluding remarks and perspectives in Section VI.

Notations: In the sequel, we use $\|Q\|=\sqrt{Q^{\top} Q}$ to denote the Euclidean norm of the given vector $Q$. Here, $I_{n} \in \mathbb{R}^{n \times n}$ denotes the unitary matrix. The notation $1_{n} \in \mathbb{R}^{n}$ is used for the column vector whose elements are 1. Given a set $\mathcal{V}$, then $|\mathcal{V}|$ denotes its cardinality. We use furthermore $Q=\left[Q_{i j}\right], \forall i \in\{1 \ldots, n\}, j \in\{1 \ldots, m\}$ to denote

$$
Q=\left[\begin{array}{ccc}
Q_{11} & \ldots & Q_{1 m} \\
\vdots & \ddots & \vdots \\
Q_{n 1} & \ldots & Q_{n m}
\end{array}\right]
$$

We use $\sigma(Q)$ to denote the sum of all elements of the matrix $Q$. Moreover, $Q \succ 0$ (or $Q \succeq 0$ ) means that $Q$ is a strictly (semi-) positive definite matrix. Similarly, $Q \prec 0$ (or $Q \preceq 0$ ) means that $Q$ is a strictly (semi-) negative definite matrix. The notation $A \otimes B$ is used for the Kronecker product of the two matrices $A$ and $B$.

\section{PROBLEM FORMULATION}

\section{A. Main assumptions}

Assumption 1. Considering a Multi-Agent System $\mathbf{\Sigma}$ composed of $N$ agents, the indices set is $\mathcal{N}=\{1, \ldots, N\}$. Each agent has its own linear discretized dynamics

$$
x_{i}(k+1)=A_{i} x_{i}(k)+B_{i} u_{i}(k), i \in \mathcal{N}
$$


with $x_{i} \in \mathbb{R}^{n}, u_{i} \in \mathbb{R}^{m}$.

Assumption 2. The agents are sharing a common working space. The position coordinates of each agent are denoted by $x_{i} \in \mathbb{R}^{n}$. It is assumed that for any point $x_{i}^{e} \in \mathbb{R}^{n}$ there exists a corresponding pair $\left(x_{i}^{e}, u_{i}^{e}\right)$ such that $\left(x_{i}^{e}, u_{i}^{e}\right)$ is a fixed point (an equilibrium point) of (1). This implies that the matrix $B_{i}$ has to be a full row rank matrix.

Assumption 3. The common working space $\mathcal{X} \subset \mathbb{R}^{n}$ is a convex bounded polyhedron.

\section{B. Main steps of the proposed approach}

At each time-instant $k$, two main steps are considered to compute the control $u_{i}(k)$ of the $i^{\text {th }}$ agent:

- STEP 1 (Partition step): Based on the available measurement of $x_{i}(k)$, a Voronoi partition ${ }^{1}$ $\mathbb{V}\left(x_{1}(k), \ldots, x_{N}(k)\right)$ will be computed.

- STEP 2 (Control step): In order to lead each agent to a fixed point (contained into its Voronoi cell), compute for each cell $\mathbb{V}_{i}(k)$ the control input $u_{i}=f_{i}\left(x_{i}, x_{j}\right), \forall j \in$ $\mathcal{N}_{i}$. The set $\mathcal{N}_{i}$ contains the indices of the neighbors whose Voronoi cells have a common facet with $\mathbb{V}_{i}(k)$.

The computation of this proposed decentralized control will be detailed in the next sections. We will start by presenting the control design based on the optimal control approach, and then extend the result by employing MPC.

\section{VORONOI-BASED OPTIMAL CONTROL DESIGN}

In this section, we propose a revisited decentralized control based on the optimal control approach, using the agent's state and the vertices of the corresponding Voronoi cell received after the partition step. Firstly, the explicit computation of the optimal solution is presented, then the stability proof is detailed by means of a resulting positive decreasing value function. In the sequel, the agent's index is neglected to simplify the notation. Let us consider an agent having its dynamics equation

$$
x(k+1)=A x(k)+B u(k)
$$

The matrix $B$ is full row rank, according to Assumption 2 . The Voronoi cell of the agent $x$ is denoted by $\mathbb{V}$, with $\mathcal{V}$ and $\mathcal{N}_{\mathcal{V}}$ representing respectively the set of its vertices $v_{i}$ and the indices set of these vertices.

\section{A. Optimal control solution}

The optimal control of (2) is the solution of the following optimization control problem

$$
\min _{u} \sum_{k=0}^{\infty} L(x(k), u(k)) \text { s.t.: } x(k+1)=A x(k)+B u(k)
$$

${ }^{1}$ Denote by $\mathbb{V}$ the Voronoi partition of a considered working space (in the Euclidian space) into $n$ cells $\mathbb{V}_{i}$, with $i=1, \ldots, n$ and $\mathbb{V}=\bigcup_{i=1}^{n} \mathbb{V}_{i}$. Notice that in the next sections, the index $i^{\text {th }}$ of each Voronoi cell will be omitted in order to simplify the presentation. with

$$
\begin{aligned}
& L(x(k), u(k))=\sum_{i, j \in \mathcal{N}_{\mathcal{V}}}\left(\left(x(k)-v_{i}\right)^{\top} Q_{i j}\left(x(k)-v_{j}\right)\right. \\
& \left.+\left(u(k)-w_{i}\right)^{\top} R_{i j}\left(u(k)-w_{j}\right)\right)
\end{aligned}
$$

indicating the running cost.

Moreover, by denoting ${ }^{2} \breve{v}=\left[\begin{array}{c}v_{1} \\ \vdots \\ v_{|\mathcal{V}|}\end{array}\right]$ and $\breve{w}=\left[\begin{array}{c}w_{1} \\ \vdots \\ w_{|\mathcal{V}|}\end{array}\right]$, we can define the augmented state $\breve{z}$ and input $\breve{t}$ as follows

$$
\begin{gathered}
\breve{z}=\left[\begin{array}{c}
x-v_{1} \\
\vdots \\
x-v_{|\mathcal{V}|}
\end{array}\right]=\breve{M} x-\breve{v} \in \mathbb{R}^{|\mathcal{V}| n} \\
\breve{t}=\left[\begin{array}{c}
u-w_{1} \\
\vdots \\
u-w_{|\mathcal{V}|}
\end{array}\right]=\breve{N} u-\breve{w} \in \mathbb{R}^{|\mathcal{V}| m}
\end{gathered}
$$

with $\breve{M}=1_{|\mathcal{V}|} \otimes I_{n}$ and $\breve{N}=1_{|\mathcal{V}|} \otimes I_{m}$.

Then the extended dynamics can be derived

$$
\breve{z}(k+1)=\breve{A} \breve{z}(k)+\breve{B} \breve{t}(k)
$$

with $\breve{A}=I_{|\mathcal{V}|} \otimes A$ and $\breve{B}=I_{|\mathcal{V}|} \otimes B$.

Let us denoting $\breve{Q}=\left[Q_{i j}\right], \breve{R}=\left[R_{i j}\right]$ with $\forall i, j \in \mathcal{N}_{\mathcal{V}}$. It requires that $\breve{Q}^{\top}=\breve{Q} \succ 0$ and $\breve{R}^{\top}=\breve{R} \succ 0$.

Theorem 1. The solution of the optimal control problem (3) for the dynamics (6) is

$$
\theta u(k)=\breve{G} \breve{w}+\breve{K} \breve{z}(k)
$$

by considering the notations

$$
\begin{aligned}
\Phi & =\breve{R}+\breve{B}^{\top} \breve{P} \breve{B} \\
\theta & =\breve{N}^{\top} \Phi \breve{N} \\
\breve{G} & =\breve{N}^{\top} \Phi \\
\breve{K} & =-\breve{N}^{\top} \breve{B}^{\top} \breve{P} \breve{A}
\end{aligned}
$$

and

$$
V(\breve{z}(k))=\breve{z}^{\top}(k) \breve{P} \breve{z}(k)
$$

which is considered as the cost-to-go of (3), with $\breve{P}=\left[P_{i j}\right]$, $\forall i, j \in \mathcal{N}_{\mathcal{V}}$ and $\breve{P}^{\top}=\breve{P} \succ 0$.

Proof. According to the Bellman principle of optimality (see [10]), the optimal control solution of (3) has to ensure

$$
V(x(k))=\min _{u(k)}\{L(x(k), u(k))+V(x(k+1))\}
$$

which leads to the following Bellman equation

$H(x(k), u(k))=L(x(k), u(k))+V(x(k+1))-V(x(k))$

Using (4) and (9), it is inferred that $H(\breve{z}(k), \breve{t}(k))=$ $\breve{z}(k)^{\top} \breve{Q} \breve{z}(k)+\breve{t}(k)^{\top} \breve{R} \breve{t}(k)+\breve{z}(k+1)^{\top} \breve{P} \breve{z}(k+1)-$ $\breve{z}(k)^{\top} \breve{P} \breve{z}(k)$. Based on (5) and (6), this can be detailed as $H(\breve{z}(k), \breve{t}(k))=\breve{z}(k)^{\top} \breve{Q} \breve{z}(k)+(\breve{N} u(k)-\breve{w})^{\top} \breve{R}(\breve{N} u(k)-$

\footnotetext{
${ }^{2}$ The ${ }^{\triangleleft}$ symbol is used to refer to all the vertices concomitantly.
} 
$\breve{w})+(\breve{A} \breve{z}(k)+\breve{B} \breve{t}(k))^{\top} \breve{P}(\breve{A} \breve{z}(k)+\breve{B} \breve{t}(k))-\breve{z}(k)^{\top} \breve{P} \breve{z}(k)$. Solving $\frac{\partial H}{\partial u}=0$ leads to $2 N^{\top} \breve{R} \breve{N} u(k)-2 N^{\top} \breve{R} \breve{w}+$ $2 \breve{N}^{\top} \breve{B}^{\top} P \breve{B} \breve{N} u(k)+2 \breve{N}^{\top} \breve{B}^{\top} \breve{P}(\breve{A} \breve{z}(k)-\breve{B} \breve{w})=0$ or equivalently $\left[\breve{N}^{\top}\left(\breve{R}+\breve{B}^{\top} \breve{P} \breve{B}\right) \breve{N}\right] u(k)=N^{\top}\left(\breve{R}+\breve{B}^{\top} \breve{P} \breve{B}\right) \breve{w}-$ $\breve{N}^{\top} \breve{B}^{\top} \breve{P} \breve{A} \breve{z}(k)$. Considering the notation (8), it leads to $\theta u(k)=\breve{G} \breve{w}+\breve{K} \breve{z}(k)$, with $\theta$ invertible.

\section{B. Stability analysis}

According to the Bellman principle of optimality, the optimal solution (7) has to ensure $H(\breve{z}(k), \breve{t}(k))=0$. It implies $L(\breve{z}(k), \breve{t}(k))+V(\breve{z}(k+1))=V(\breve{z}(k))$. In order to guarantee the Lyapunov stability, a sufficient condition is $V(\breve{z}(k+1))-V(\breve{z}(k))=-L(\breve{z}(k), \breve{t}(k)) \leq 0$.

Starting from $L(\breve{z}(k), \breve{t}(k))+V(\breve{z}(k+1))=$ $\breve{z}(k)^{\top} \breve{Q} \breve{z}(k)+\breve{t}(k)^{\top} \breve{R} \breve{t}(k)+\breve{z}(k+1)^{\top} \breve{P} \breve{z}(k+1)$, it can be derived that $L(\breve{z}(k), \breve{t}(k))+V(\breve{z}(k+1))=$ $\breve{z}(k)^{\top} \breve{Q} \breve{z}(k)+(\breve{N} u(k)-\breve{w})^{\top} \breve{R}(\breve{N} u(k)-\breve{w})+$ $(\breve{A} \breve{z}(k)+\breve{B} \breve{N} u(k)-\breve{B} \breve{w})^{\top} \breve{P}(\breve{A} \breve{z}(k)+\breve{B} \breve{N} u(k)-\breve{B} \breve{w})$. Using the notations (8a), (8b), (8c) and (8d) then replacing $u(k)$ from (7), with $\theta$ invertible, this becomes $L(\breve{z}(k), \breve{t}(k))+V(\breve{z}(k+1))=\breve{z}^{\top}(k)(\breve{Q}+$ $\left.\breve{A}^{\top} \breve{P} \breve{A}\right) \breve{z}(k)+\breve{w}^{\top} \Phi \breve{w}-(\breve{G} \breve{w}+\breve{K} \breve{z}(k))^{\top} \theta^{-\top} \theta \theta^{-1}(\breve{G} \breve{w}+$ $\breve{K} \breve{z}(k))-2 \breve{w}^{\top} \breve{B}^{\top} \breve{P} \breve{A} \breve{z}(k)$. Regrouping the terms in $\breve{z}(k)$ and $\breve{w}$, we obtain $L(\breve{z}(k), \breve{t}(k))+V(\breve{z}(k+1))=$ $\breve{z}^{\top}(k)\left(\breve{Q}+\breve{A}^{\top} \breve{P} \breve{A}-\breve{K}^{\top} \theta^{-\top} \breve{K}\right) \breve{z}(k)+\breve{w}^{\top}(\Phi-$ $\left.\breve{G}^{\top} \theta^{-\top} \breve{G}\right) \breve{w}-2 \breve{w}^{\top}\left(\breve{B}^{\top} \breve{P} \breve{A}+\breve{G}^{\top} \theta^{-\top} \breve{K}\right) \breve{z}(k)$. However $L(\breve{z}(k), \breve{t}(k))+V(\breve{z}(k+1))=V(\breve{z}(k))$ requires that the two terms $\breve{w}^{\top}\left(\Phi-\breve{G}^{\top} \theta^{-\top} \breve{G}\right) \breve{w}$ and $\breve{w}^{\top}\left(\breve{B}^{\top} \breve{P} \breve{A}+\breve{G}^{\top} \theta^{-\top} \breve{K}\right)$ have to vanish. For a non zero vector $\breve{w}$, it is difficult to impose $\breve{w}^{\top}\left(\Phi-\breve{G}^{\top} \theta^{-\top} \breve{G}\right) \breve{w}=0$. A lightened condition $\breve{w}^{\top}\left(\Phi-\breve{G}^{\top} \theta^{-\top} \breve{G}\right) \breve{w} \leq 0$ together with $\breve{w}^{\top}\left(\breve{B}^{\top} \breve{P} \breve{A}+\breve{G}^{\top} \theta^{-\top} \breve{K}\right)=0$ are further considered, leading to $L(\breve{z}(k), \breve{t}(k))+V(\breve{z}(k+1)) \leq V(\breve{z}(k))$. In this context, the following theorem formulates sufficient conditions.

Theorem 2. If there exists a vector $\tilde{w}$ such as $\tilde{w} \in \mathbb{R}^{m}$ and

$$
\begin{aligned}
& \breve{G} \breve{w}=\breve{G} \breve{N} \tilde{w} \\
& \breve{w}^{\top} \breve{B}^{\top} \breve{P} \breve{A}=-\tilde{w}^{\top} \breve{K} \\
& {\left[\begin{array}{cc}
\Phi & \breve{G}^{\top} \\
\breve{G} & \theta
\end{array}\right] \preceq 0}
\end{aligned}
$$

then the following expressions are verified $\breve{w}^{\top}(\Phi-$ $\left.\breve{G}^{\top} \theta^{-\top} \breve{G}\right) \breve{w} \leq 0$ and $\breve{w}^{\top}\left(\breve{B}^{\top} \breve{P} \breve{A}+\breve{G}^{\top} \theta^{-\top} \breve{K}\right)=0$.

Proof. Applying the Schur complement in (12c) leads to $\Phi-$ $\breve{G}^{\top} \theta^{-\top} G \preceq 0$. Multiplying left and right by the non zero vector $\breve{w}^{\top}$ and $\breve{w}$ leads to $\breve{w}^{\top}\left(\Phi-\breve{G}^{\top} \theta^{-\top} \breve{G}\right) \breve{w} \leq 0$.

Using (12a), the expression $\breve{w}^{\top}\left(\breve{B}^{\top} \breve{P} \breve{A}+\right.$ $\left.\breve{G}^{\top} \theta^{-\top} \breve{K}\right)=\breve{w}^{\top} \breve{B}^{\top} \breve{P} \breve{A}+\breve{w}^{\top} \breve{G}^{\top} \theta^{-\top} \breve{K}$ becomes $\breve{w}^{\top} \breve{B}^{\top} \breve{P} \breve{A}+\tilde{w}^{\top} \breve{N}^{\top} \breve{G}^{\top} \theta^{-\top} \breve{K}$. From (8b) and (8c), it results in $\breve{G} \breve{N}=\theta$. This allows us to obtain the following result $\breve{w}^{\top}\left(\breve{B}^{\top} \breve{P} \breve{A}+\breve{G}^{\top} \theta^{-\top} \breve{K}\right)=\breve{w}^{\top} \breve{B}^{\top} \breve{P} \breve{A}+\tilde{w}^{\top} \theta^{\top} \theta^{-\top} \breve{K}$. Using (12b), this leads to $\breve{w}^{\top}\left(\breve{B}^{\top} \breve{P} \breve{A}+\breve{G}^{\top} \theta^{-\top} \breve{K}\right)=0$.
Following Theorem 2, if the solution (12) is feasible then there exists a vector $\tilde{w}$ to guarantee $L(\breve{z}(k), \breve{t}(k))+V(\breve{z}(k+$ $1)) \leq V(\breve{z}(k))$. However, a feasible vector $\tilde{w}$ depends on the choice of the weighting matrices $\breve{P}, \breve{Q}$ and $\breve{R}$, which will be illustrated via the following proposition.

Proposition 1. Consider the matrices $R \in \mathbb{R}^{m \times m}, P \in$ $\mathbb{R}^{n \times n}$ such as $R=R^{\top} \succ 0, P=P^{\top} \succ 0$, and a weighting structure matrix $\Delta \in \mathbb{R}^{|\mathcal{V}| \times|\mathcal{V}|}$. Hence the matrices $\breve{R}$ and $\breve{P}$ can be chosen such that $\breve{R}=\Delta \otimes R$ and $\breve{P}=\Delta \otimes P$. The matrix $\breve{Q}$ can be obtained by solving a Riccati equation.

Proof. Consider a weighting structure matrix $\Delta=$ $\left[\Delta_{1} \ldots \Delta_{|\mathcal{V}|}\right]$, with $\Delta_{i}$ denoting the $i^{\text {th }}$ column of $\Delta$. The equation (12a) is rewritten as $\breve{N}^{\top} \Phi \breve{w}=\breve{N}^{\top} \Phi \breve{N} \tilde{w}$. Due to (8a), we get $\Phi=\breve{R}+\breve{B}^{\top} \breve{P} \breve{B}=\Delta \otimes(R+$ $\left.B^{\top} P B\right)$. Hence (12a) becomes $\breve{N}^{\top}\left(\Delta \otimes\left(R+B^{\top} P B\right)\right) \breve{w}=$ $\breve{N}^{\top}\left(\Delta \otimes\left(R+B^{\top} P B\right)\right) \breve{N} \tilde{w}$. From the definition of $\breve{N}$, it is infered that $N^{\top}=\left[\begin{array}{lll}I_{m} & \ldots & I_{m}\end{array}\right] \in \mathbb{R}^{m \times(m|\mathcal{V}|)}$. Rewriting $\Delta=\left[\delta_{i j}\right], \forall i, j \in\{1, \ldots,|\mathcal{V}|\}$ and denoting $\tilde{R}=R+B^{\top} P B$ leads to $\left[\sum_{j=1}^{|\mathcal{V}|} \delta_{j, 1} \tilde{R} \quad \ldots \quad \sum_{j=1}^{|\mathcal{V}|} \delta_{j,|\mathcal{V}|} \tilde{R}\right] \breve{w}=$ $\left[\begin{array}{llll}\sum_{j=1}^{|\mathcal{V}|} \delta_{j, 1} \tilde{R} & \ldots & \sum_{j=1}^{|\mathcal{V}|} \delta_{j,|\mathcal{V}|} \tilde{R}\end{array}\right]\left[\begin{array}{lll}I_{m} & \ldots & I_{m}\end{array}\right]^{\top} \tilde{w}$. It is possible to write $\sum_{i=1}^{|\mathcal{V}|} \sigma\left(\Delta_{i}\right) \tilde{R} w_{i}=\sigma(\Delta) \tilde{R} \tilde{w}$, with the scalars $\sigma\left(\Delta_{i}\right)=\sum_{j=1}^{|\mathcal{V}|} \delta_{j, i}$ and $\sigma(\Delta)$ is the sum of all the elements of $\Delta$. After symplifying $\tilde{R}$, we obtain $\sum_{i=1}^{|\mathcal{V}|} \sigma\left(\Delta_{i}\right) w_{i}=\sigma(\Delta) \tilde{w}$, which is further equivalent to $\tilde{w}=\sum_{i=1}^{|\mathcal{V}|}(\sigma(\Delta))^{-1} \sigma\left(\Delta_{i}\right) w_{i}$. Similar results will be obtained for (12b). In (12c), because all matrices $\Phi, \breve{G}$ and $\theta$ depend on $\Delta$, we can obtain a value for $\Delta$ by solving the feasibility problem (12c) with the decision variable $\Delta$. Therefore, we can conclude that all conditions (12) are fulfilled proving a choice for $\tilde{w}$.

Using Theorem 2 with Proposition 1 ensures the closedloop stability of (6) with respect to the optimal control solution (7). We can see in the sequel that it implies also the closed-loop stability of (2) by proving the equivalence between the Lyapunov stability conditions of these two dynamics.

Replacing (5b) in (6) and then using (7) yields

$$
\breve{z}(k+1)=\left(\breve{A}+\breve{B} \breve{N} \theta^{-1} \breve{K}\right) \breve{z}(k)+\breve{B} \breve{N} \theta^{-1} \breve{G} \breve{w}-\breve{B} \breve{w}
$$

with the Lyapunov stability condition

$$
\left(\breve{A}+\breve{B} \breve{N} \theta^{-1} \breve{K}\right)^{\top} \breve{P}\left(\breve{A}+\breve{B} \breve{N} \theta^{-1} \breve{K}\right) \preceq \breve{P}
$$

Furthermore, substituting (7) in the dynamics equation (2) and using (5a) to replace $\breve{z}$ by $x$, we get

$$
x(k+1)=\left(A+B \theta^{-1} \breve{K} \breve{M}\right) x(k)+B \theta^{-1}(\breve{G} \breve{w}-\breve{K} \breve{v})
$$


which leads to the Lyapunov stability condition of (2)

$$
\left(A+B \theta^{-1} \breve{K} \breve{M}\right)^{\top} P\left(A+B \theta^{-1} \breve{K} \breve{M}\right) \preceq P
$$

Now consider the two dynamics equation (2) and (6) with the stability conditions (16) and (14), respectively. The following theorem shows the equivalence of the stability of these two dynamics.

Theorem 3. The expression (16) is verified if and only if (14) is verified.

Proof. Consider (14). Left and right multiplying (14) respectively with the matrix $\breve{M}^{\top}$ and $\vec{M}$, we obtain $\breve{M}^{\top}\left(\breve{A}+\breve{B} \breve{N} \theta^{-1} \breve{K}\right)^{\top} \breve{P}\left(\breve{A}+\breve{B} \breve{N} \theta^{-1} \breve{K}\right) \breve{M} \preceq$ $\breve{M}^{\top} \breve{P} \breve{M}$. From the definitions of $\breve{A}$ and $\breve{M}$ it is inferred that $\breve{A} \breve{M}=\breve{M} A$ and $\breve{B} \breve{N}=\breve{M B}$. Thus we get $\breve{M}^{\top}\left(\breve{A}+\breve{B} \breve{N} \theta^{-1} \breve{K}\right)^{\top} \breve{P}\left(\breve{A}+\breve{B} \breve{N} \theta^{-1} \breve{K}\right) \breve{M}=$ $\left(A+B \theta^{-1} \breve{K} \breve{M}\right)^{\top} \breve{M}^{\top} \breve{P} \breve{M}\left(A+B \theta^{-1} \breve{K} \breve{M}\right)$, thus $(A+$ $\left.B \theta^{-1} \breve{K} \breve{M}\right)^{\top} \breve{M}^{\top} \breve{P} \breve{M}\left(A+B \theta^{-1} \breve{K} \breve{M}\right) \preceq \breve{M}^{\top} \breve{P} \breve{M}$. Вy choosing a matrix $P=\breve{M}^{\top} \breve{P} \breve{M}$, we get the condition (16).

Considering (16) in order to prove (14) can be done in a similar way.

The previuos theorem illustrated the equivalence between the stability of systems (2) and (6). The next step is to find the equilibrium point the agent's state $x(k)$ converges to. The following theorem is used to determine this point.

Proposition 2. If the dynamics equation (2) is stabilized w.r.t the control (7), $x(k)$ and $u(k)$ of (2) converge asymptotically towards an equilibrium point $(\bar{x}, \bar{u})$ determined as:

$$
\begin{aligned}
& \left(I-A-B \theta^{-1} \breve{K} \breve{M}\right) \bar{x}=B \theta^{-1}(\breve{G} \breve{w}-\breve{K} \breve{v}) \\
& (I-A) \bar{x}=B \bar{u}
\end{aligned}
$$

In fact, finding the equilibrium points for the systems (2) and (15) leads to expressions (17).

We prove above that the stability of (2) is equivalent to the stability of (6), additionally it is certain that the agent's state and input converge to the equilibrium point $(\bar{x}, \bar{u})$ determined via (17). However until now the role of the vector $\tilde{w}$ introduced in Theorem 2 is still not clarified. We need to know if there exist some relations between the equilibrium point $(\bar{x}, \bar{u})$ and the vector $\tilde{w}$.

Remark 1. By using (12a), the right-hand side of (17a) becomes $B \theta^{-1} \breve{G} \breve{N} \tilde{w}-B \theta^{-1} \breve{K} \breve{v}$. Using (8b) and (8c) leads to $\theta=\breve{G N}$, thus $\left(I-A-B \theta^{-1} \breve{K} \breve{M}\right) \bar{x}=B \tilde{w}-B \theta^{-1} \breve{K} \breve{v}$. Substituting (17b) in the left-hand side of (17a) leads to $B \bar{u}-B \theta^{-1} \breve{K} \breve{M} \bar{x}=B \tilde{w}-B \theta^{-1} \breve{K} \breve{v}$. After term by term identification on the two sides of this result, we find

$$
\begin{array}{r}
\breve{K} \breve{M} \bar{x}=\breve{K} \breve{v} \\
\bar{u}=\tilde{w}
\end{array}
$$

The following proposition illustrates the location of the fixed point $(\bar{x}, \bar{u})$ of the agent relative to its Voronoi cell $\mathbb{V}$.

Proposition 3. $(\bar{x}, \bar{u})$ determined by solving (17) belongs to the interior of $\mathbb{V}$, depending on the choice of $\Delta$.

Proof. Replacing the notation (8d) into (18a) yields $\breve{N}^{\top} \breve{B}^{\top} \breve{P} \breve{A} \breve{M}=\breve{N}^{\top} \breve{B}^{\top} \breve{P} \breve{A} \breve{v}$. Using Proposition 1, one has $\breve{B}^{\top} \breve{P} \breve{A}=\Delta \otimes\left(B^{\top} P A\right)$ thus $\breve{N}^{\top}\left(\Delta \otimes\left(B^{\top} P A\right)\right) \breve{M} \bar{x}=$ $\breve{N}^{\top}\left(\Delta \otimes\left(B^{\top} P A\right)\right) \breve{v}$. Silimar to the proof of Proposition 1 , we get $\sigma(\Delta) \bar{x}=\sum_{i=1}^{|\mathcal{V}|} \sigma\left(\Delta_{i}\right) v_{i}$ then $\bar{x}=$ $\sum_{i=1}^{|\mathcal{V}|}(\sigma(\Delta))^{-1} \sigma\left(\Delta_{i}\right) v_{i}$. Due to $\sum_{i=1}^{|\mathcal{V}|} \sigma\left(\Delta_{i}\right)=\sigma(\Delta)$, we obtain $\sum_{i=1}^{|\mathcal{V}|}(\sigma(\Delta))^{-1} \sigma\left(\Delta_{i}\right)=1$ and finally we get $\bar{x} \in \operatorname{int}(\mathbb{V})$.

To conclude, the stability of (2) w.r.t the control (7) is proved. This control ensures the convergence of the agent's state $x$ towards the fixed point $\bar{x}$ inside its Voronoi cell $\mathbb{V}$, according to Proposition 3. These results will be extended next within the Model Predictive Control context.

\section{VORONOI-BASED MPC CONTROL DESIGN}

The results above can be extended over a finite horizon $N_{p}$. Solving an unconstrained MPC problem is similar to solving an optimal control problem over a finite horizon (see [11]). We will show in this section the explicit solution of this control problem and then prove that the convergence can be built similarly as the optimal control approach. For brevity, we use $k=0$ to denote the current instant time.

\section{A. Explicit control solution}

The decentralized MPC framework is

$$
\begin{aligned}
& u(0)=\arg \min _{u(k)} \sum_{k=0}^{N_{p}-1} L(x(k), u(k))+V\left(x\left(N_{p}\right)\right) \\
& \text { s.t: } x(k+1)=A x(k)+B u(k)
\end{aligned}
$$

The cost function in the MPC framework (19) is $J=$ $N_{p}-1$ $\sum_{k=0}^{p} L(x(k), u(k))+V\left(x\left(N_{p}\right)\right)$. Notice that the running cost $L(x(k), u(k))$ and the terminal cost function $V(x(k))$ are defined similar to (4) and (9) respectively. Here, $V\left(x\left(N_{p}\right)\right)$ denotes the final state penalty expected to be reached at the end of the prediction horizon.

By reusing the notations $\breve{P}, \breve{Q}, \breve{R}, \breve{A}, \breve{B}, \breve{M}, \breve{N}, \breve{v}, \breve{w}$ and the extended dynamics equation (6) of the previous section, we can derive the following result by a recurrent construction over the prediction horizon $N_{p}$

$$
\begin{gathered}
\mathbf{z}=\mathbf{A} \breve{z}(0)+\mathbf{B}(\mathbf{N} \mathbf{U}-\mathbf{w}) \\
\text { with } \mathbf{z}=\left[\begin{array}{c}
\breve{z}(1) \\
\vdots \\
\breve{z}\left(N_{p}\right)
\end{array}\right], \mathbf{U}=\left[\begin{array}{c}
u(0) \\
\vdots \\
u\left(N_{p}-1\right)
\end{array}\right], \mathbf{w}=\left[\begin{array}{c}
\breve{w} \\
\vdots \\
\breve{w}
\end{array}\right], \mathbf{A}= \\
{\left[\begin{array}{c}
\breve{A} \\
\vdots \\
\breve{A}^{N_{p}}
\end{array}\right], \mathbf{B}=\left[\begin{array}{ccc}
\breve{N} & & \\
\vdots & \ddots & \\
\breve{A}^{N_{p}-1} \breve{B} & \ldots & \breve{B}
\end{array}\right], \mathbf{N}=\left[\begin{array}{ccc} 
& & \\
& \ddots & \\
& & \breve{N}
\end{array}\right]}
\end{gathered}
$$


Then the cost function is rewritten as

$$
\begin{aligned}
& J=\breve{z}(0)^{\top} \breve{Q} \breve{z}(0) \\
& +(\mathbf{A} \breve{z}(0)+\mathbf{B N U}-\mathbf{B w})^{\top} \mathbf{P}(\mathbf{A} \breve{z}(0)+\mathbf{B N U}-\mathbf{B w}) \\
& +\mathbf{U}^{\top} \mathbf{N}^{\top} \mathbf{R} \mathbf{N U}-2 \mathbf{U}^{\top} \mathbf{N}^{\top} \mathbf{R} \mathbf{w}+\mathbf{w}^{\top} \mathbf{R w}
\end{aligned}
$$

with $\mathbf{P}=\left[\begin{array}{cccc}\breve{Q} & & & \\ & \ddots & & \\ & & \breve{Q} & \\ & & & \breve{P}\end{array}\right], \mathbf{R}=\left[\begin{array}{ccc}\breve{R} & & \\ & \ddots & \\ & & \breve{R}\end{array}\right]$.

In order to simplify the presentation, consider the notation

$$
\begin{aligned}
\mathbf{\Phi} & =\mathbf{R}+\mathbf{B}^{\top} \mathbf{P B} \\
\mathbf{\Theta} & =\mathbf{N}^{\top} \mathbf{\Phi} \mathbf{N} \\
\mathbf{G} & =\mathbf{N}^{\top} \mathbf{\Phi} \\
\mathbf{K} & =-\mathbf{N}^{\top} \mathbf{B}^{\top} \mathbf{P A}
\end{aligned}
$$

Theorem 4. Consider the optimization control problem (19). The optimal control solution over the prediction horizon $N_{p}$ of this problem is

$$
\mathbf{\Theta} \mathbf{U}=\mathbf{G} \mathbf{w}+\mathbf{K} \breve{z}(0)
$$

Proof. The proof is identical to the optimal control case (see the proof of Theorem 1)

\section{B. Stability analysis}

Replacing (23) in (21), we get $J=\breve{z}(0)^{\top}\left(\breve{Q}+\mathbf{A}^{\top} \mathbf{P A}-\right.$ $\left.\mathbf{K}^{\top} \boldsymbol{\Theta}^{-\top} \mathbf{K}\right) \breve{z}(0)$

$+\mathbf{w}^{\top}\left(\boldsymbol{\Phi}-\mathbf{G}^{\top} \boldsymbol{\Theta}^{-\top} \mathbf{G}\right) \mathbf{w}-2 \mathbf{w}^{\top}\left(\mathbf{B}^{\top} \mathbf{P A}+\mathbf{G}^{\top} \mathbf{\Theta}^{-\top} \mathbf{K}\right) \breve{z}(0)$. Similar to optimal control approach, the control (23) guarantees $J \leq J^{*}$, with $J^{*}=\breve{z}(0)^{\top} \breve{P} \breve{z}(0)$ if and only if $\mathbf{w}^{\top}(\boldsymbol{\Phi}-$ $\left.\mathbf{G}^{\top} \boldsymbol{\Theta}^{-\top} \mathbf{G}\right) \mathbf{w} \leq 0$ and $\mathbf{w}^{\top}\left(\mathbf{B}^{\top} \mathbf{P A}+\mathbf{G}^{\top} \boldsymbol{\Theta}^{-\top} \mathbf{K}\right)=0$. This result is further formulated via the Theorem 5 .

Theorem 5. If there exists a vector $\mathbf{w} \in \mathbb{R}^{m N_{p}}$ which respects the conditions

$$
\begin{aligned}
& \mathbf{G w}=\mathbf{G N} \tilde{\mathbf{w}} \\
& \mathbf{w}^{\top} \mathbf{B}^{\top} \mathbf{P A}=-\tilde{\mathbf{w}}^{\top} \mathbf{K} \\
& {\left[\begin{array}{cc}
\mathbf{\Phi} & \mathbf{G}^{\top} \\
\mathbf{G} & \boldsymbol{\Theta}
\end{array}\right] \preceq 0}
\end{aligned}
$$

then the following expressions are verified $\mathbf{w}^{\top}(\mathbf{\Phi}-$ $\left.\mathbf{G}^{\top} \boldsymbol{\Theta}^{-\top} \mathbf{G}\right) \mathbf{w} \leq 0$ and $\mathbf{w}^{\top}\left(\mathbf{B}^{\top} \mathbf{P A}+\mathbf{G}^{\top} \boldsymbol{\Theta}^{-\top} \mathbf{K}\right)=0$.

Proof. The proof is identical to the optimal control case (see Theorem 2).

Applying Theorem 5 helps us to keep $J \leq J^{*}$ and therefore the stability is guaranteed at the end of the prediction horizon, i.e. $\sum_{k=0}^{N_{p}-1} L(x(k), u(k))+V\left(x\left(N_{p}\right)\right) \leq V(x(0))$ leads to $V\left(x\left(N_{p}\right)\right)-V(x(0)) \leq-\sum_{k=0}^{N_{p}-1} L(x(k), u(k))$. In the following, we will use the weighting matrices $\breve{P}, \breve{Q}, \breve{R}$ from the previous section in order to guarantee $V(x)$ $1))-V(x(k)) \leq-L(x(k), u(k))$. Rewriting this over the prediction horizon $N_{p}$, we derive that

$$
\begin{aligned}
V(x(1))-V(x(0)) & \leq-L(x(0), u(0)) \\
V(x(2))-V(x(1)) & \leq-L(x(1), u(1)) \\
& \vdots \\
V\left(x\left(N_{p}\right)\right)-V\left(x\left(N_{p}-1\right)\right) & \leq-L\left(x\left(N_{p}-1\right), u\left(N_{p}-1\right)\right)
\end{aligned}
$$

By taking the sum of these inequalities above, we get $V\left(x\left(N_{p}\right)\right)-V(x(0)) \leq-\sum_{k=0}^{N_{p}-1} L(x(k), u(k))$. In other words, the stability of the MPC problem (19) is covered by the stability of the optimal control problem (3).

\section{Simulation Results}

Two simulation scenarios will be presented. The considered MAS $\Sigma$ contains $N_{a}=15$ homogeneous agents. The agent dynamics is

$$
x_{i}(k+1)=x_{i}(k)+T_{s} u_{i}(k), i \in \mathcal{N}
$$

with $T_{s}=0.1$ and $x_{i}, u_{i} \in \mathbb{R}^{2}$ being the position and the speed. The steady-state equation of (25) is $(I-$ I) $x_{i}=T_{s} u_{i}$, hence all positions in $\mathbb{R}^{2}$ can be a fixed point of (25) if the speed is zero. The working region is $\mathcal{X}=\operatorname{Conv}\{(-20,-20),(20,-20),(20,20),(-20,20)\}$. These choices of the dynamics equation and $\mathcal{X}$ satisfy all the Assumptions 1, 2 and 3. We will study respectively the performance of the control design (3) and (19) via the movement of the agents over $\mathcal{X}$ and also the tracking distance $\left\|x_{i}-\bar{x}_{i}\right\|$. In Figs. 1 and 3 , we use red colored circles, green colored stars and blue colored circle-lines to denote respectively the initial positions $x_{i}(0)$, the evolution of fixed point $\bar{x}_{i}(k)$ and $x_{i}(k)$. The next step consists in finding the matrices $P, Q, R$. We can choose for instance $R=\left[\begin{array}{ll}0.2 & 0.1 \\ 0.1 & 0.1\end{array}\right]$. The matrix $P=\left[\begin{array}{ll}9.84 & 1.24 \\ 1.24 & 8.74\end{array}\right]$ is found such that $\left(I+T_{s} K\right)^{\top} P\left(I+T_{s} K\right) \preceq P$, with the feedback gain $K$ obtained by pole placement techniques (with 0.8 and 0.5 as stable poles). The matrix $Q=\left[\begin{array}{cc}2.74 & -0.26 \\ -0.26 & 4.06\end{array}\right]$ is computed by substituting $P$ and $R$ in a Riccati equation. From $P, Q, R$, the matrices $\breve{Q}, \breve{R}$ and $\breve{P}$ are obtained and are further used in the optimal control problem (3). Here we choose $\Delta=I_{|\mathcal{V}|}$

In the first scenario, the decentralized optimal control (3) is used. The results are illustrated in Fig. 1. All the agents from their initial positions deploy $\mathcal{X}$ and quickly obtain the optimal coverage. This is shown in Fig. 2 where the tracking errors $\left\|x_{i}-\bar{x}_{i}\right\|$ drop to zero after a certain time.

We get similar results in the second scenario (see Figs. 34), using the unconstrained MPC control (19). In this case, we use the same matrices $P, Q, R$ and $\Delta$ of the first scenario. The prediction horizon is $N_{p}=3$. 


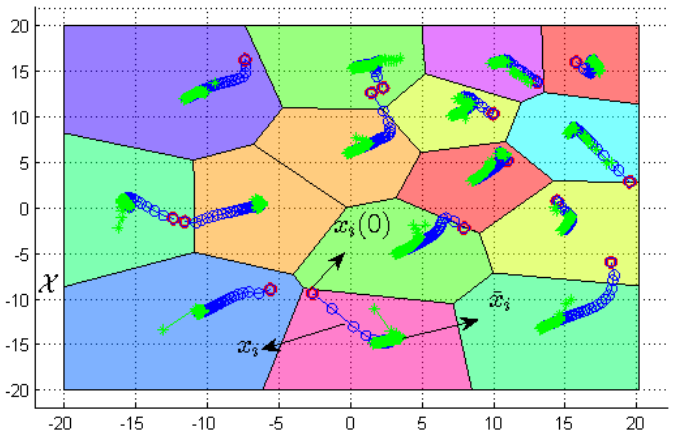

Fig. 1. Coverage by using optimal control approach.

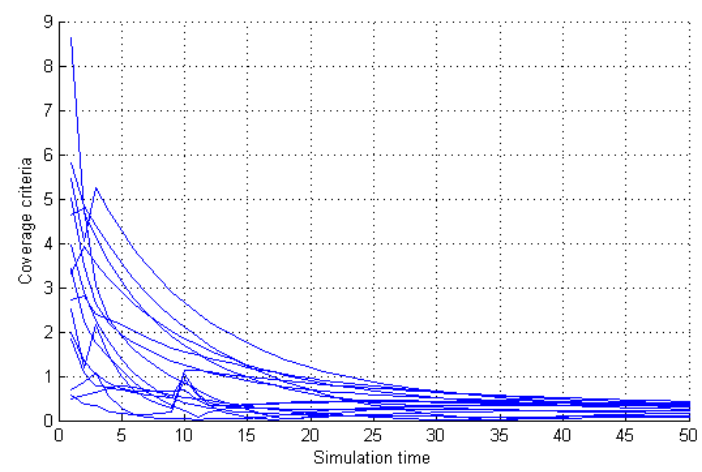

Fig. 2. Coverage criteria by using optimal control approach.

\section{CONCLUSION}

This paper revisits the decentralized coverage problem by addressing respectively the optimal control approach and MPC approach. The main idea consists of providing the decentralized control policies to enhance the coverage of a group of cooperative homogeneous mobile agents over a bounded region. The classical Voronoi algorithm is employed to partition the whole region in real time. This partition step is based on the positions of the agents and subsequently gives the necessary information to design the decentralized control action for each agent. Both proposed approaches use the current state of the agent and the vertices of its Voronoi cell to compute the decentralized control. The constrained MPC case as well as the robustness analysis with respect to different uncertainties will be considered in future work.

\section{ACKNOWLEDGEMENT}

The authors gratefully acknowledge the discussion with Prof. Luis Rodrigues and Michael Di Perna (on topics related to the optimal coverage problems [12]) and with Prof. Sorin Olaru (on topics related to predictive control for Multi-Agent systems). The first author acknowledges the support of the LIA Project between L2S and SYTACom.

\section{REFERENCES}

[1] G. Lafferriere, J. Caughman, and A. Williams, "Graph theoretic methods in the stability of vehicle formations," in IEEE American Control Conference, vol. 4, 2004, pp. 3729-3734.

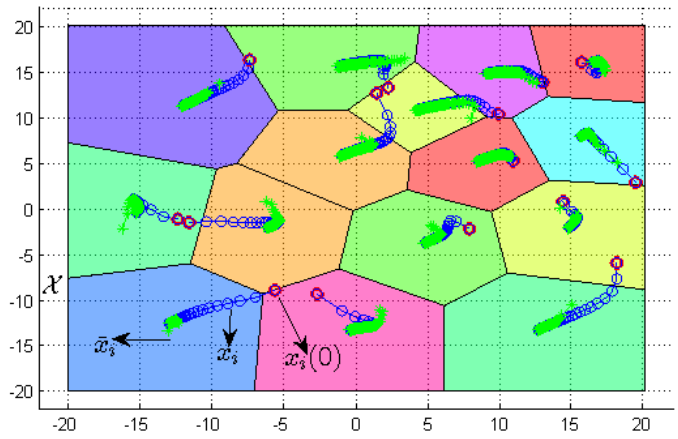

Fig. 3. Coverage by using unconstrained MPC approach.

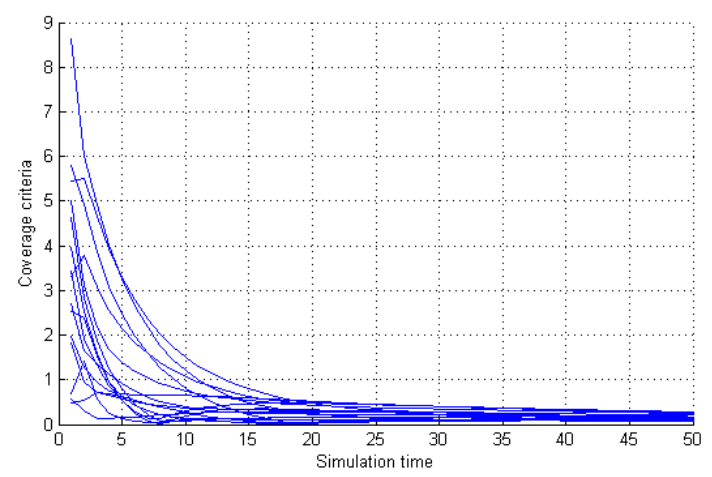

Fig. 4. Coverage criteria by using unconstrained MPC approach.

[2] H. G. Tanner, A. Jadbabaie, and G. J. Pappas, "Flocking in teams of nonholonomic agents," in Cooperative Control. Springer, 2005, pp. 229-239.

[3] G. Bitsoris and S. Olaru, "Further results on the linear constrained regulation problem," in Mediterranean Control Conference, Greece, 2013.

[4] I. Prodan, "Commande sous contraintes de systemes dynamiques multi-agents," Ph.D. thesis, Supelec (in English), 2012.

[5] E. Kontouras, A. Tzes, and L. Dritsas, "Adversary control strategies for discrete-time systems," in ECC, 2014, pp. 2508-2513.

[6] S. Stankovic, M. Stanojevic, and D. Siljak, "Decentralized overlapping control of a platoon of vehicles," Control Systems Technology, IEEE Transactions on, vol. 8, no. 5, pp. 816-832, Sep 2000.

[7] F. A. Fontes, D. B. Fontes, and A. C. Caldeira, "Model predictive control of vehicle formations," in Optimization and Cooperative Control Strategies. Springer, 2009, pp. 371-384.

[8] M. T. Nguyen, C. Stoica Maniu, and S. Olaru, "Control invariant partition for heterogeneous multi-agent dynamical systems," in $19^{t h}$ International Conference on System Theory, Control and Computing, Cheile Gradistei, Romania, 2015.

[9] R. M. Murray, "Recent research in cooperative control of multivehicle systems," Journal of Dynamic Systems, Measurement, and Control, vol. 129, no. 5, pp. 571-583, 2007.

[10] R. Bellman, "Dynamic programming and a new formalism in the calculus of variations," Proceedings of the national academy of sciences, vol. 40, no. 4, pp. 231-235, 1954.

[11] C. E. Garcia, D. M. Prett, and M. Morari, "Model predictive control: theory and practicea survey," Automatica, vol. 25, no. 3, pp. 335-348, 1989.

[12] M. Moarref and L. Rodrigues, "An optimal control approach to decentralized energy-efficient coverage problems," in IFAC World Congress, Cape Town, South Africa, 2014. 